\title{
Conversion of natural forest results in a significant degradation of soil hydraulic properties in the highlands of Kenya
}

S.O. Owuor $^{\mathrm{a}, \mathrm{b}}$

K. Butterbach-Bahla, b, *

klaus.butterbach-bahl@kit.edu

A.C. Guzha

S. Jacobs ${ }^{a, d}$

L. Merbold ${ }^{\mathrm{b}}$

M.C. Rufino ${ }^{\mathrm{d}, \mathrm{e}}$

D.E. Pelster

E. Díaz-Pinés ${ }^{\mathrm{a}, \mathrm{f}}$

L. Breuer ${ }^{g, h}$

aInstitute of Meteorology and Climate Research, Atmospheric Environmental Research (IMK-IFU), Karlsruhe Institute of Technology (KIT), Kreuzeckbahnstr. 19, 82467 Garmisch-Partenkirchen, Germany

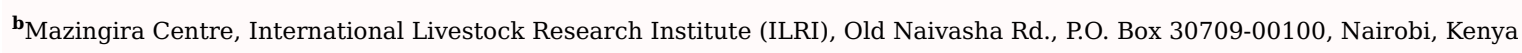

${ }^{\mathbf{c}}$ United States Forest Service, c/o CIFOR, World Agroforestry Centre, United Nations Avenue Gigiri, P.O. Box 30677-00100, Nairobi, Kenya

${ }^{\mathbf{d}}$ Center for International Forestry Research (CIFOR), C/o World Agroforestry Centre, United Nations Avenue Gigiri, P.O. Box 30677-00100, Nairobi, Kenya

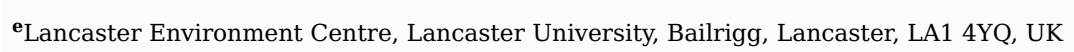

Institute of Soil Research, University of Natural Resources and Life Sciences (BOKU), Peter-Jordan-Strasse 82, 1190 Vienna, Austria

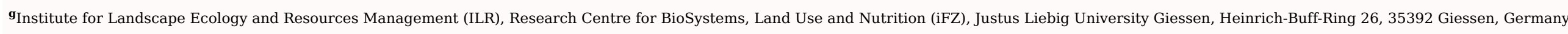

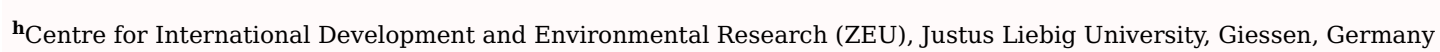

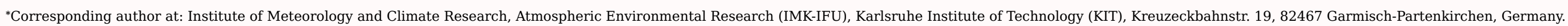

\section{Abstract}

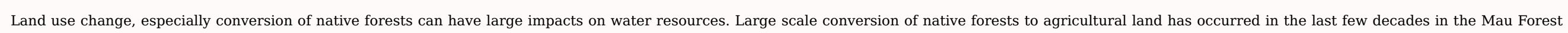

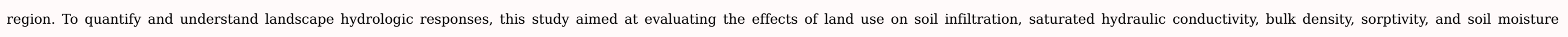

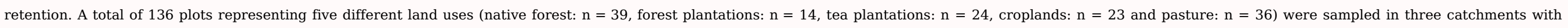

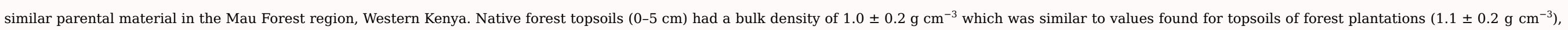

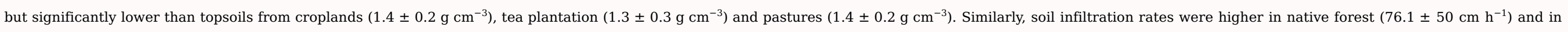

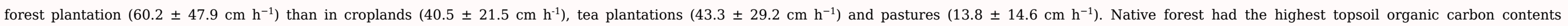

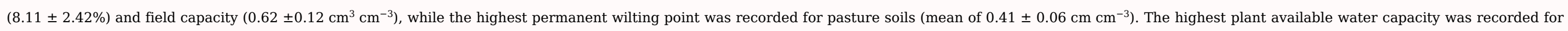




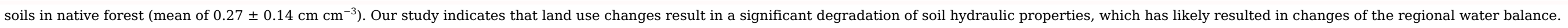

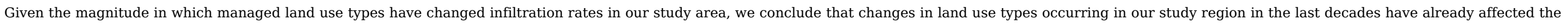

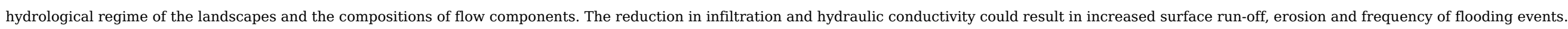

Keywords: Infiltration rates; Water retention characteristics; Double ring infiltrometer; South West Mau; Land use change

\section{Introduction}

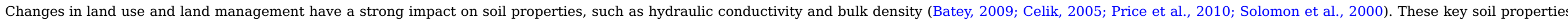

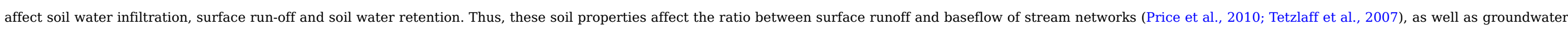

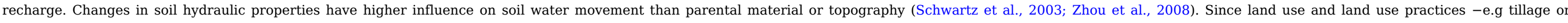

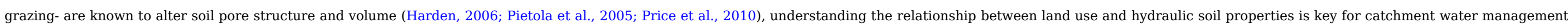
(Minasny and George, 1999).

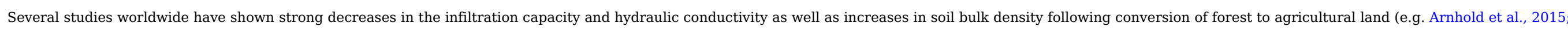

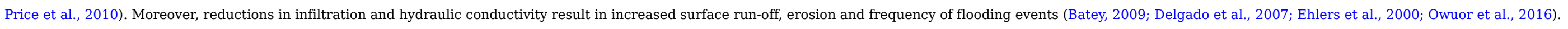

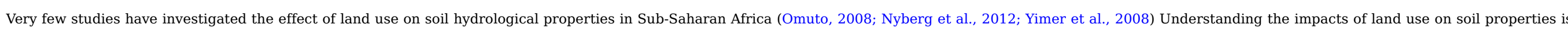

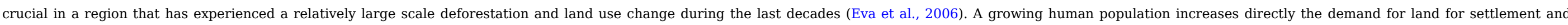

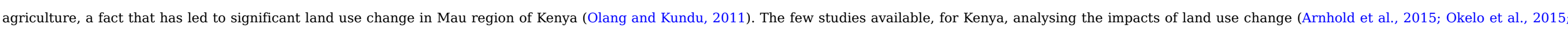

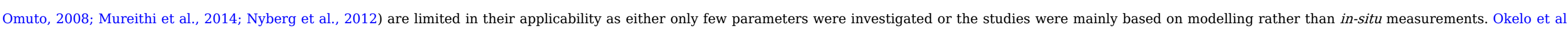

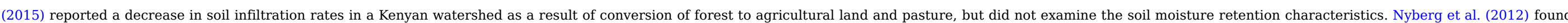

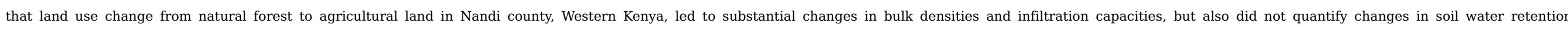

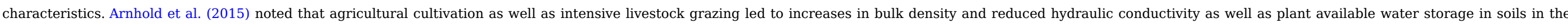

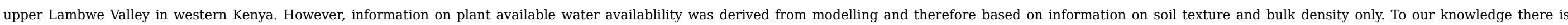

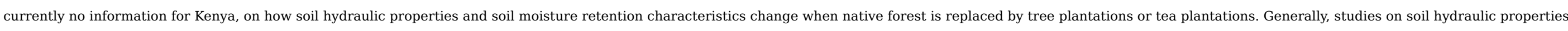
and moisture retention characteristics on African montane forests is conspicously missing

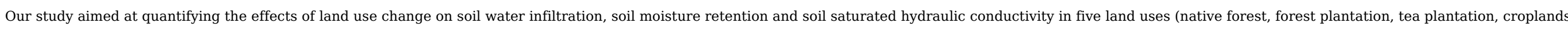

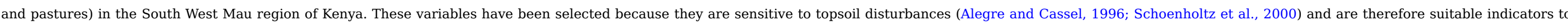

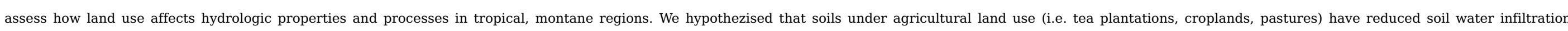
capacity, volumetric moisture content and increased bulk density compared to forests (i.e. native forest and forest plantations).

\section{Materials and methods}

\subsection{Site description and experimental design}

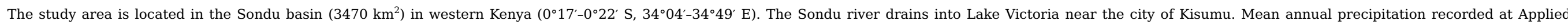

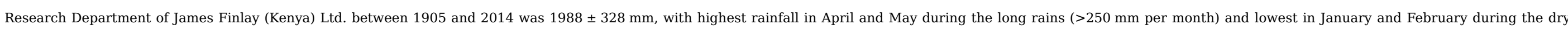

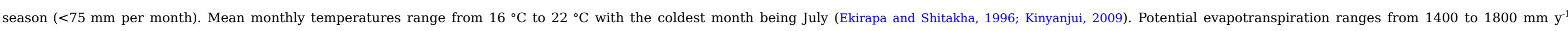
(Kinyanjui, 2009).

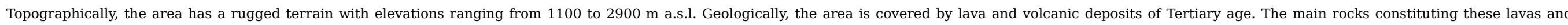




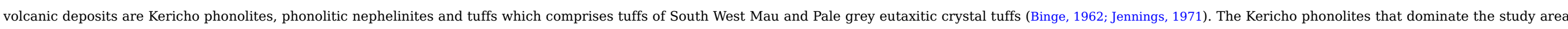

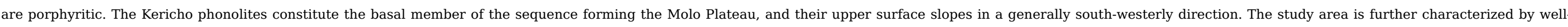

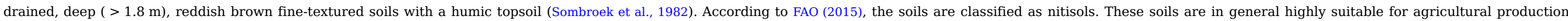
(Sombroek et al., 1982).

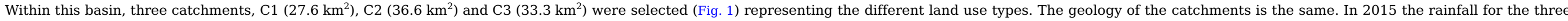

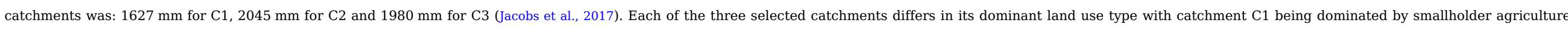

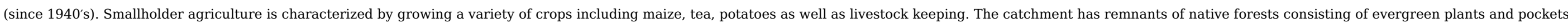

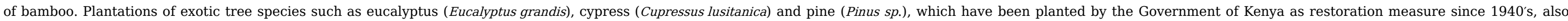
exist in the catchment. Groundwater is the major source of water supply for the residents. 

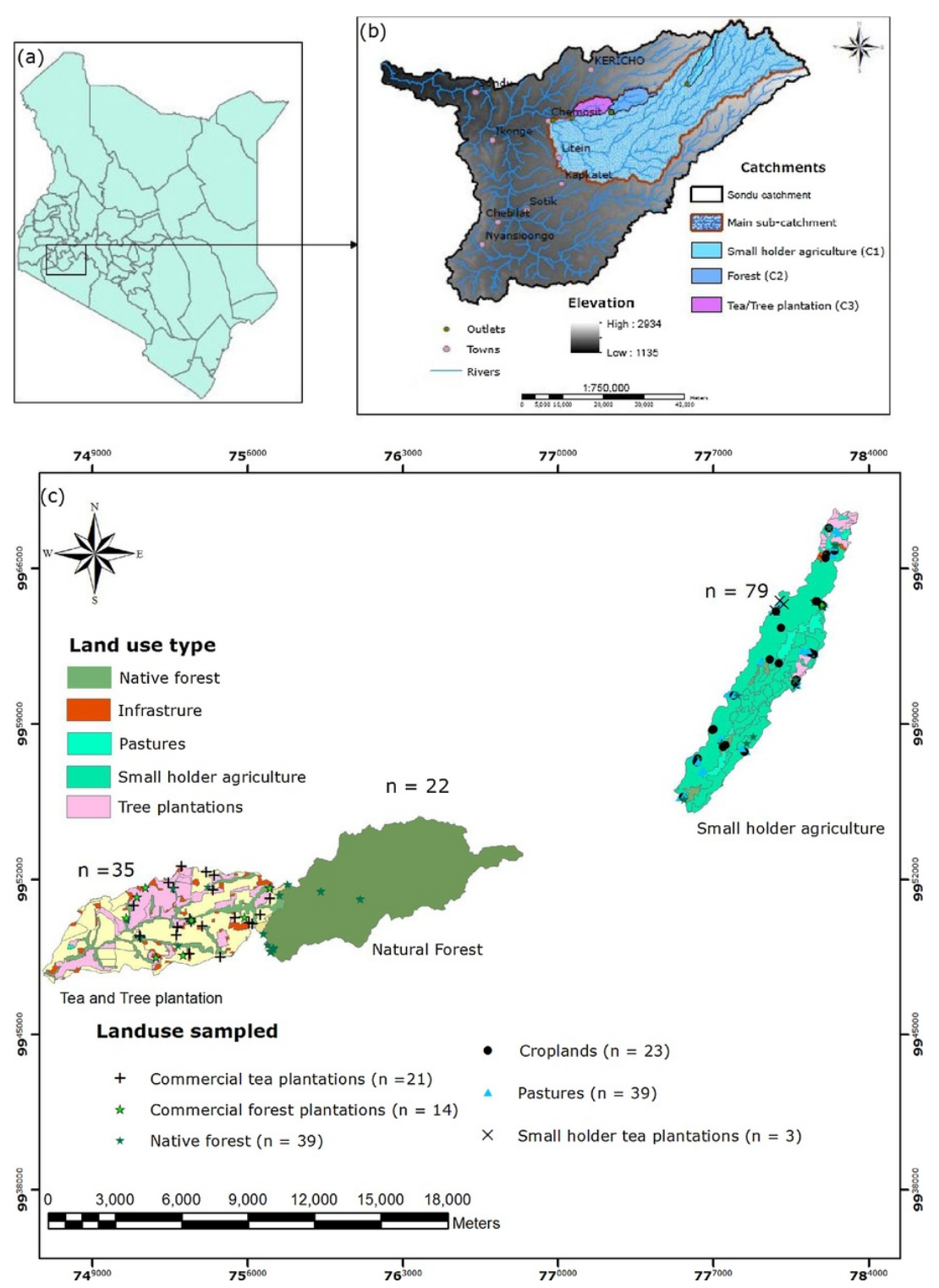

Fig. 1 Location of the study area and sampling plots. (a) Map of Kenya showing location of South West Mau. (b) Extent of Sondu basin. (c) Location of the sampling plots. alt-text: Fig. 1

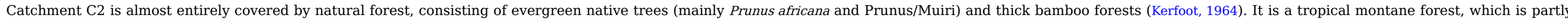
degraded through livestock grazing, illegal logging and charcoal burning.

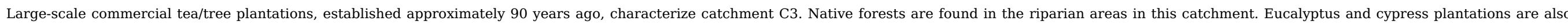
found in the catchment and are used to provide firewood for the tea factories. 


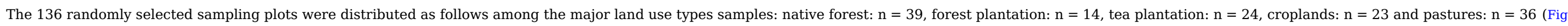

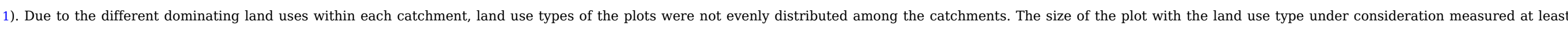

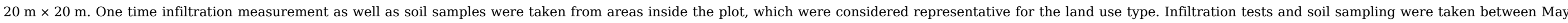
and July 2015.

\subsection{Field measurements}

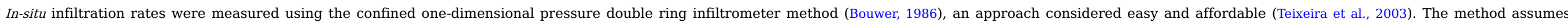

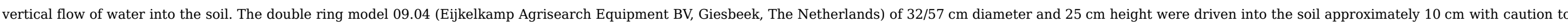

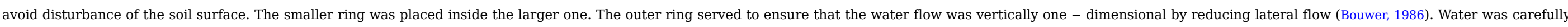

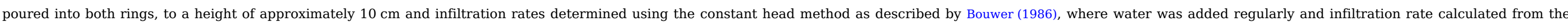

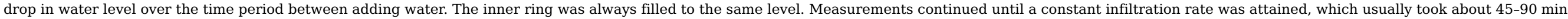

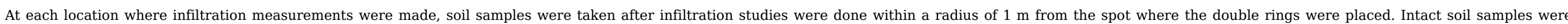

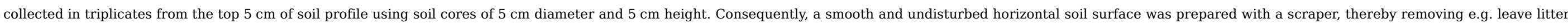

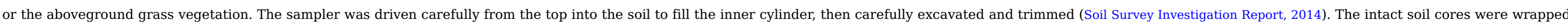

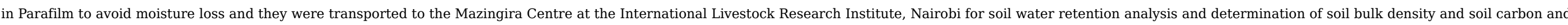

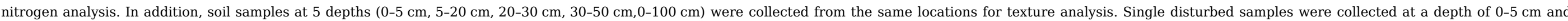

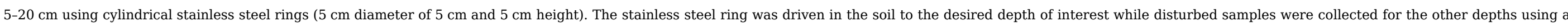
soil auger. All the samples were packed, labeled and transported to the laboratory for texture analysis. The soil from 5 to $20 \mathrm{~cm}$ depth were also used for determination of bulk density as well.

\subsection{Laboratory measurements}

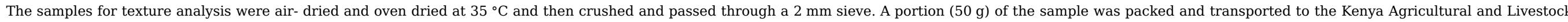

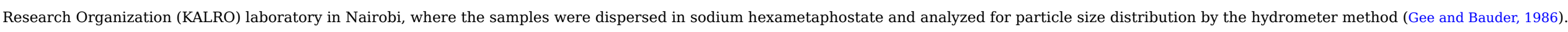

\subsection{Estimation of soil hydraulic properties}

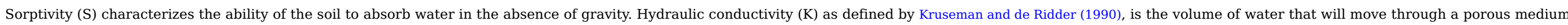

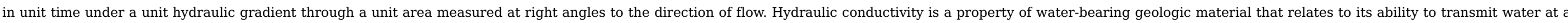
standard temperature and density (Sterrett, 2007).

For every infiltration measurement, sorptivity and hydraulic conductivity were calculated by optimizing Philip's equation (1957; Eq. (1)) in R version 3.22 (R Core Team, 2015, see Appendix A). $i(t)=\frac{1}{2} S t^{1 / 2}+K$

where $i$ is infiltration rate $\left(\mathrm{cm} \mathrm{h}^{-1}\right), t$ is time for infiltration, $S$ is the sorptivity $\left(\mathrm{cm} \mathrm{h}^{-1 / 2}\right)$, and $K$ is hydraulic conductivity $\left(\mathrm{cm} \mathrm{h}^{-1}\right)$.

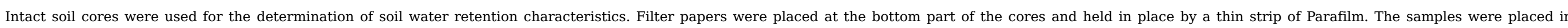

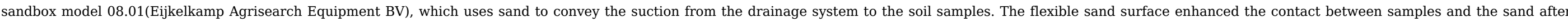

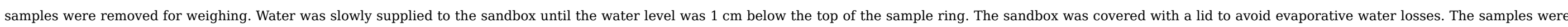

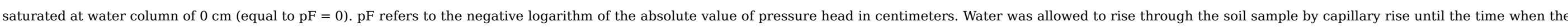

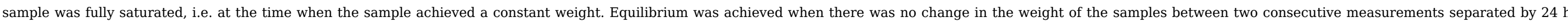

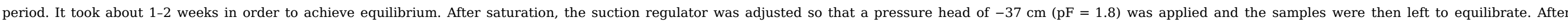




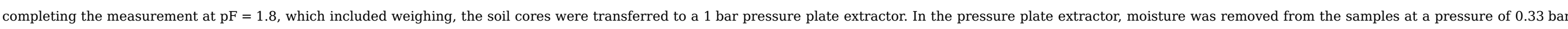

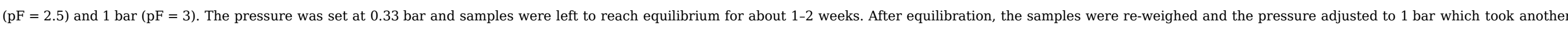

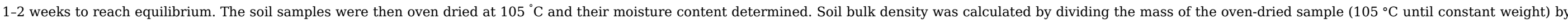

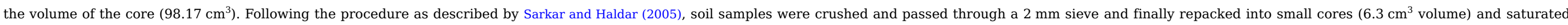

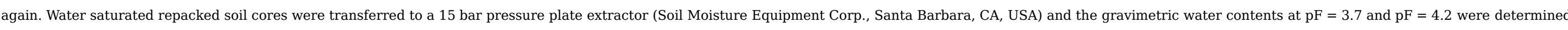

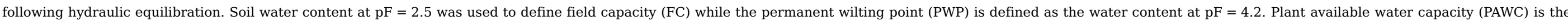
water stored between $\mathrm{pF}=2.5$ and $\mathrm{pF}=4.2$

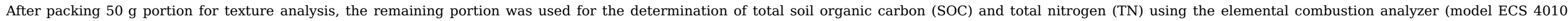

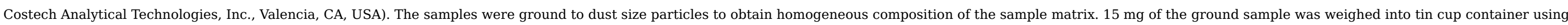
analytical micro-balance and forceps. The weighed samples were loaded in the elemental combustion analyzer automatic sampler for analysis.

\subsection{Statistical analysis}

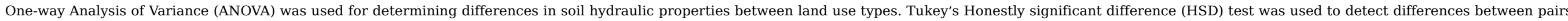

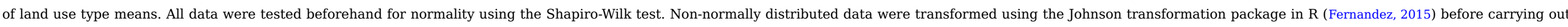

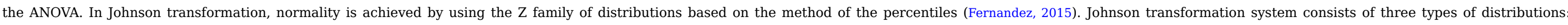
bounded system (SB), log-normal system (SL) and unbounded system (SU). These systems are described by the equations (2-4) respectively.

Boundedform $(S U): Y=\gamma+\eta \log \left(\frac{\chi-\varepsilon}{\lambda+\varepsilon-\chi}\right)$

Lognormal form $(S B): Y=\gamma+\eta \log \left(\frac{\chi-\varepsilon}{\lambda}\right)$

Unboundedform $(S U): Y=\gamma+\eta \sinh ^{-1}\left(\frac{\chi-\varepsilon}{\lambda}\right)$

Where:

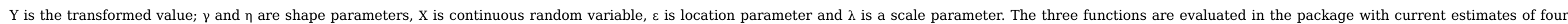
parameters. The four parameters are optimized until one of the three transformation functions produces the best normality test result.

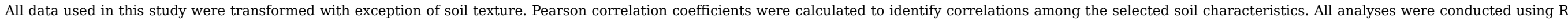
3.22 ( $R$ Core Team, 2015) at a significance level of $\mathrm{P} \leq 0.05$.

\section{Results}

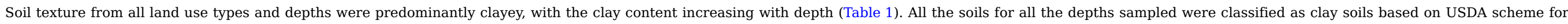
soil classification. On average, soils from the lower depths $(50-100 \mathrm{~cm}$ ) contained slightly less sand and silt and slightly higher clay content (Table 1).

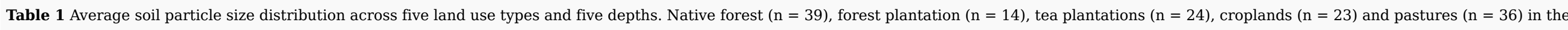
South West Mau region, Kenya.

alt-text: Table 1

Land use

Depth $(\mathrm{cm})$

Sand (\%)

Clay (\%)

Native forest

$0-5$

5-20
17.8

17.7
62.5

63.3 


\begin{tabular}{|c|c|c|c|}
\hline & $20-30$ & 16.6 & 65.2 \\
\hline & $30-50$ & 13.4 & 68.2 \\
\hline & $50-100$ & 14.5 & 69.0 \\
\hline \multirow[t]{5}{*}{ Forest plantation } & $0-5$ & 23.1 & 52.1 \\
\hline & $5-20$ & 19.5 & 56.1 \\
\hline & $20-30$ & 19.4 & 57.4 \\
\hline & $30-50$ & 17.3 & 61 \\
\hline & $50-100$ & 14.9 & 64.9 \\
\hline \multirow[t]{5}{*}{ Tea plantation } & $0-5$ & 15.6 & 64.3 \\
\hline & $5-20$ & 13.6 & 67.1 \\
\hline & $20-30$ & 13.9 & 67.7 \\
\hline & $30-50$ & 12.3 & 71 \\
\hline & $50-100$ & 11.3 & 74.4 \\
\hline \multirow[t]{5}{*}{ Croplands } & $0-5$ & 19.8 & 54.8 \\
\hline & $5-20$ & 19.3 & 55.3 \\
\hline & $20-30$ & 18.3 & 56.7 \\
\hline & $30-50$ & 16.3 & 62 \\
\hline & $50-100$ & 15.9 & 64.7 \\
\hline \multirow[t]{5}{*}{ Pastures } & $0-5$ & 21.1 & 53.8 \\
\hline & $5-20$ & 18.6 & 56.7 \\
\hline & $20-30$ & 17.2 & 60.2 \\
\hline & $30-50$ & 14.5 & 62.9 \\
\hline & $50-100$ & 14.5 & 67.3 \\
\hline
\end{tabular}

Native forest topsoil $(0-5 \mathrm{~cm})$ had a mean ( \pm standard deviation) bulk density of $1.0 \pm 0.2 \mathrm{~g} \mathrm{~cm}^{-3}$, which was similar to that of topsoils taken from forest plantation $\left(1.1 \pm 0.2 \mathrm{~g} \mathrm{~cm}{ }^{-3}\right)$. The mean bulk density for native forest and forest plantation were lower $(P<0.001)$ than the bulk density values for croplands $\left(1.4 \pm 0.2 \mathrm{~g} \mathrm{~cm}^{-3}\right)$, tea plantations $\left(1.3 \pm 0.3 \mathrm{~g} \mathrm{~cm}^{-3}\right)$, and pastures $\left(1.4 \pm 0.2 \mathrm{~g} \mathrm{~cm}{ }^{-3}\right)$ (Fig. 2$)$. A tendency towards lower bulk density for soils taken from native forest system compared to those from agricultural land uses could also be detected for the $5-20 \mathrm{~cm}$ soil layer though this trend across land use types was not significant $(P=0.168)$. 


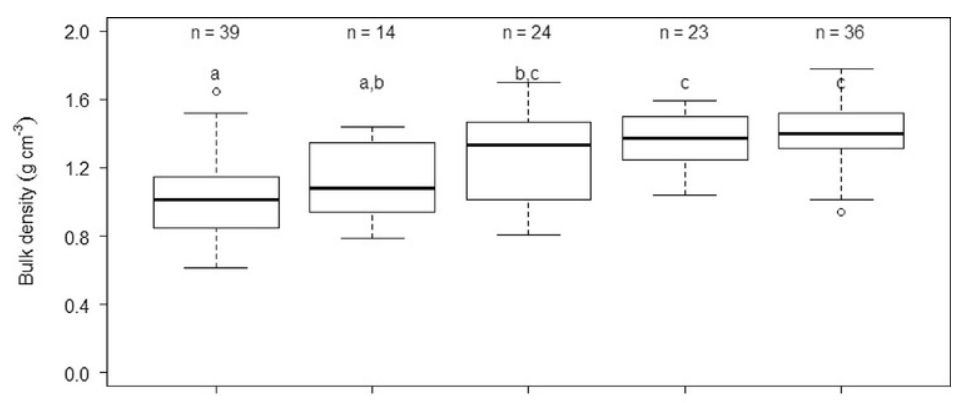

Native forest Forest plantations Tea plantations Croplands Pastures

Land use type

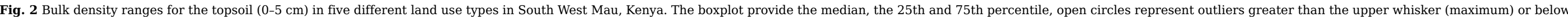
the lower whisker (minimum). Different letters denote significant differences $(P<0.05)$.

\section{alt-text: Fig. 2}

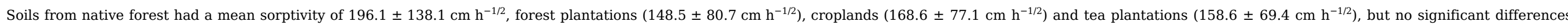
among those land uses were found. However, the sorptivity for the pasture soils was lower than all the other soils $\left(55.9 \pm 30.3 \mathrm{~cm} \mathrm{~h}^{-1 / 2} ; P<0.001\right)$

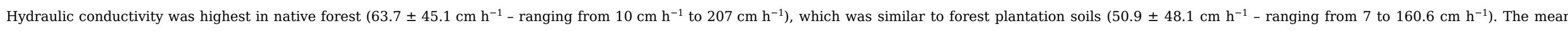

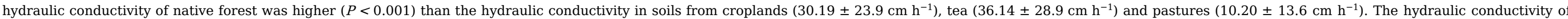
croplands ranged from $4 \mathrm{~cm} \mathrm{~h}^{-1}$ to $81 \mathrm{~cm} \mathrm{~h}^{-1}$, tea plantations from 8 to $90.1 \mathrm{~cm} \mathrm{~h}^{-1}$ and pastures from 2 to $53 \mathrm{~cm} \mathrm{~h}^{-1}$.

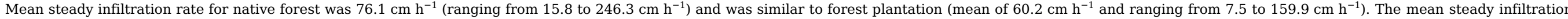

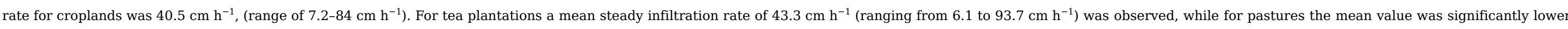
with $13.8 \mathrm{~cm} \mathrm{~h}^{-1}$ (ranging from 1.2 to $60.3 \mathrm{~cm} \mathrm{~h}^{-1}$ ) (Table 2).

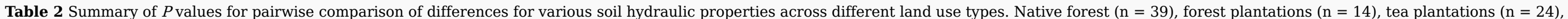
croplands $(\mathrm{n}=23)$ and pastures $(\mathrm{n}=36)$ in the South West Mau region, Kenya.

\begin{tabular}{|c|c|c|c|c|c|}
\hline Land use type & $\rho$ Bulk density $(0-5 \mathrm{~cm})$ & $\rho$ Bulk density $(5-20 \mathrm{~cm})$ & Sorptivity $\left(\mathrm{cm} \mathrm{h}^{-1 / 2}\right)$ & Hydraulic conductivity $\left(\mathrm{cm} \mathrm{h}^{-1}\right)$ & Steady infiltration rate $\left(\mathrm{cm} \mathrm{h}^{-1}\right)$ \\
\hline Pastures - Croplands & 0.9 & 1 & $<0.001$ & 0.03 & $<0.001$ \\
\hline Native forest - Croplands & $<0.001$ & 0.6 & 1 & 0.001 & 0.01 \\
\hline Forest plantation - Croplands & 0.03 & 1 & 0.8 & 0.4 & 0.8 \\
\hline Tea plantation - Croplands & 0.7 & 1 & 1 & 0.9 & 1 \\
\hline Native forest - Pastures & $<0.001$ & 0.2 & $<0.001$ & $<0.001$ & $<0.001$ \\
\hline Forest plantation - Pastures & 0.002 & 1 & $<0.001$ & $<0.001$ & $<0.001$ \\
\hline Tea plantation - Pastures & 0.1 & 1 & $<0.001$ & 0.001 & $<0.001$ \\
\hline Forest plantation - Native forest & 0.6 & 0.9 & 0.54 & 0.6 & 0.5 \\
\hline Tea plantation - Native forest & $<0.001$ & 0.2 & 0.9 & 0.03 & 0.008 \\
\hline
\end{tabular}


Native forest topsoils showed the highest field capacity $\left(0.62 \pm 0.12 \mathrm{~cm} \mathrm{~cm}^{-1}\right)$, the highest wilting point $\left(0.35 \pm 0.7 \mathrm{~cm} \mathrm{~cm}^{-1}\right)$, and the highest plant available water capacity $\left(0.27 \pm 0.14 \mathrm{~cm} \mathrm{~cm}^{-1}\right)($ Table 3$)$.

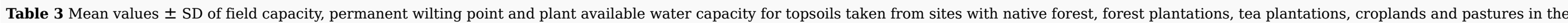
South West Mau. Different letters down the columns denotes significant differences $(p<0.05)$. $p F$ is defined as the negative logarithm of the absolute value of pressure head in centimeters. alt-text: Table 3

\begin{tabular}{|c|c|c|c|}
\hline Land use type & Field capacity $(\mathrm{pF} 2.5)\left(\mathrm{cm} \mathrm{cm}^{-3}\right)$ & Permanent wilting point $(\mathrm{pF} 4.2)\left(\mathrm{cm} \mathrm{cm}^{-3}\right)$ & Plant available water capacity $(\mathrm{pF} 2.5-4.2)\left(\mathrm{cm} \mathrm{cm}^{-3}\right)$ \\
\hline Native forest $(n=39)$ & $0.62 \pm 0.12$ a & $0.35 \pm 0.073 \mathrm{a}$ & $0.27 \pm 0.14$ a \\
\hline Forest plantation $(\mathrm{n}=14)$ & $0.47 \pm 0.058 \mathrm{a}, \mathrm{b}$ & $0.32 \pm 0.01$ a & $0.16 \pm 0.11 \mathrm{a}, \mathrm{b}$ \\
\hline Tea plantation $(\mathrm{n}=24)$ & $0.44 \pm 0.058 \mathrm{~b}$ & $0.29 \pm 0.079$ a & $0.14 \pm 0.1 \mathrm{~b}$ \\
\hline Croplands $(n=23)$ & $0.47 \pm 0.059 \mathrm{~b}$ & $0.37 \pm 0.044$ a & $0.09 \pm 0.08 \mathrm{~b}$ \\
\hline Pastures $(\mathrm{n}=36)$ & $0.54 \pm 0.088 b$ & $0.41 \pm 0.06$ b & $0.14 \pm 0.1 \mathrm{~b}$ \\
\hline
\end{tabular}

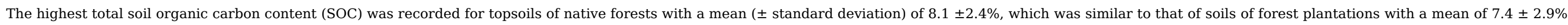

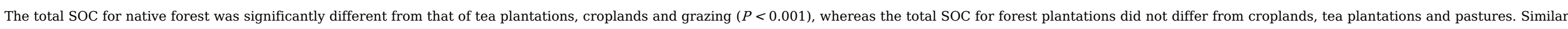
trends were observed for total nitrogen (TN). Table 4 shows a summary of total SOC and TN for every land use type.

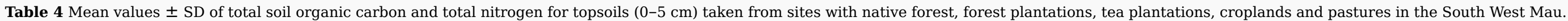
Different letters down the columns denotes significant differences $(\mathrm{p}<0.05)$

\section{alt-text: Table 4}

\begin{tabular}{|c|c|c|}
\hline Land use type & Total soil organic carbon (\%) & Total nitrogen (\%) \\
\hline Native forest $(n=25)$ & $8.11 \pm 2.42$ a & $0.49 \pm 0.23^{a}$ \\
\hline Forest plantation $(\mathrm{n}=7)$ & $7.40 \pm 2.92 \mathrm{ab}$ & $0.33 \pm 0.27^{a, b}$ \\
\hline Tea plantation $(\mathrm{n}=20)$ & $5.84 \pm 1.33 b$ & $0.19 \pm 0.099 \mathrm{~b}$ \\
\hline Croplands ( $\mathrm{n}=19$ ) & $5.69 \pm 1.11 \mathrm{~b}$ & $0.21 \pm 0.12^{\mathrm{b}}$ \\
\hline Pastures $(\mathrm{n}=28)$ & $6.20 \pm 0.92 b$ & $0.18 \pm 0.099 b$ \\
\hline
\end{tabular}

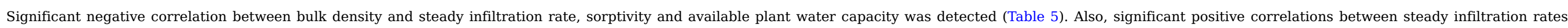
sorptivity and saturated hydraulic conductivity was found. No correlation was detected between plant available water capacity and sorptivity or steady infiltration rate.

Table 5 Pearson Correlations between the soil individual hydraulic parameters from the South West Mau region, Kenya.

alt-text: Table 5

\begin{tabular}{|c|c|c|c|c|c|c|c|}
\hline & Bulk density $(0-5 \mathrm{~cm})$ & Saturated hydraulic conductivity & Plant-available water capacity & Sorptivity & Steady infiltration rate & Total soil organic carbon & Total nitrogen \\
\hline Bulk density $(0-5 \mathrm{~cm})$ & 1.00 & & & & & & \\
\hline Saturated hydraulic conductivity & $-0.43^{* * *}$ & 1.00 & & & & & \\
\hline
\end{tabular}




\begin{tabular}{|l|l|}
\hline Plant available water capacity & $-0.43^{* * *}$ \\
\hline Sorptivity & $-0.28^{* *}$ \\
\hline Steady infiltration rate & $-0.47^{* * *}$ \\
\hline Total organic carbon & $-0.69^{* * *}$ \\
\hline Total nitrogen & $-0.44^{* *}$ \\
\hline
\end{tabular}

\begin{tabular}{|l|}
\hline 0.03 \\
\hline $0.48^{* * *}$ \\
\hline $0.92^{* * *}$ \\
\hline $0.33^{* *}$ \\
\hline $0.36^{*}$ \\
\hline
\end{tabular}

\begin{tabular}{|l|l|l|l|}
\hline 1.00 & & \\
\hline 0.006 & 1.00 & \\
\hline 0.05 & $0.69 * * *$ & 1.00 \\
\hline $0.52^{* * *}$ & 0.03 & 0.27 \\
\hline 0.13 & 0.19 & $0.34^{*}$ \\
\hline
\end{tabular}

Significance levels: $* P<0.05, * * P<0.01, * * * P<0.001$.

\section{Discussion}

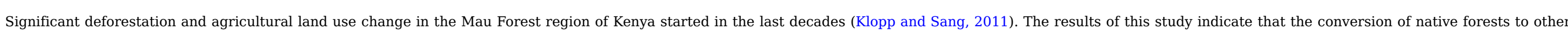
land use types, resulted in significant changes in soil hydraulic properties and water retention characteristics.

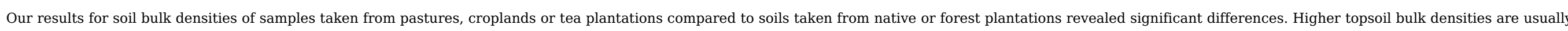

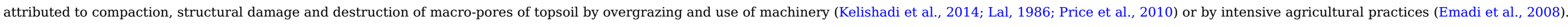

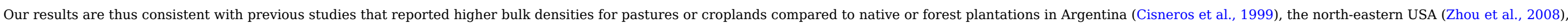
Costa Rica (Reiners et al., 1994), Iraq (Emadi et al., 2008) and Ethiopia (Selassie and Ayanna, 2013).

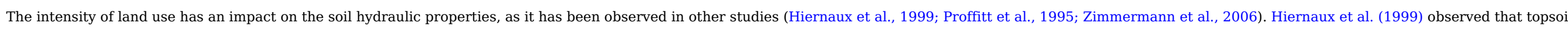
compaction was due to intensive, i.e. continuous grazing for nine months per year in Niger.

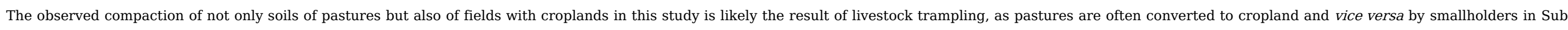
Saharan Africa. In contrast, the soil compaction observed for soils of tea plantation is likely the result of site preparation and manual harvesting (Hamza and Anderson, 2005).

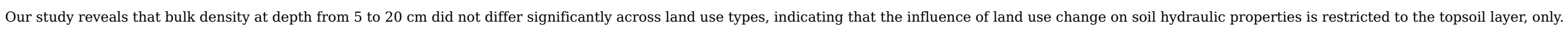

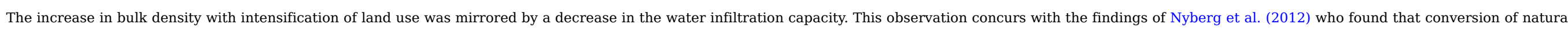

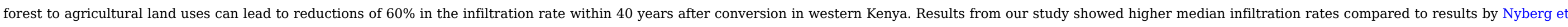

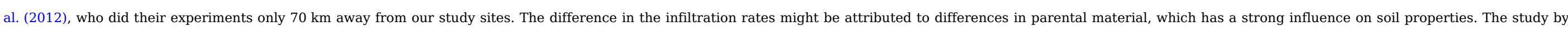

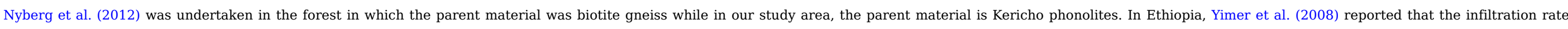

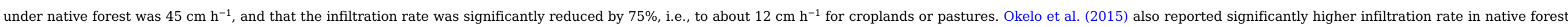

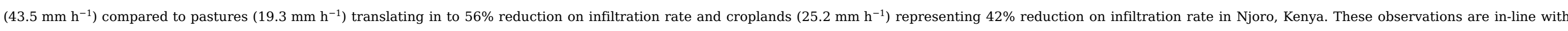

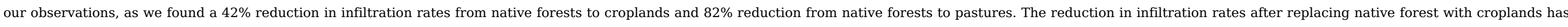

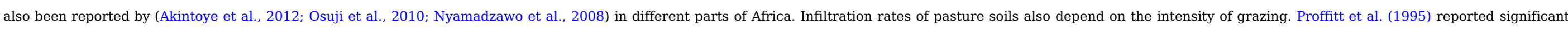

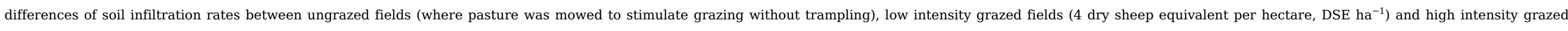
fields (8 DSE ha ${ }^{-1}$ ), which reduced by $51 \%$ and $58 \%$, respectively, in well-structured soils over a period of ten months at Merredin in Western Australia.

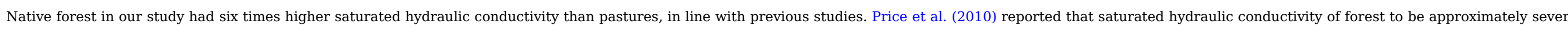

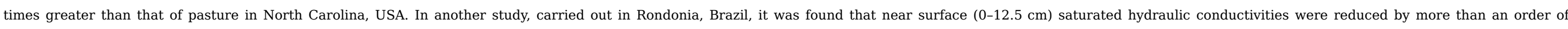

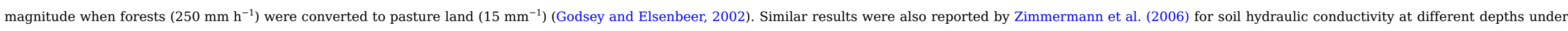

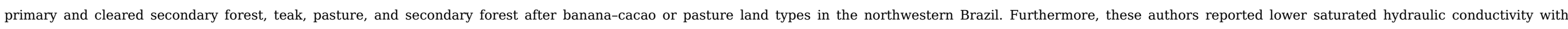
increasing land use intensity. 


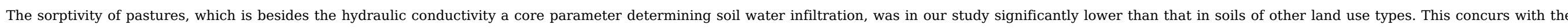

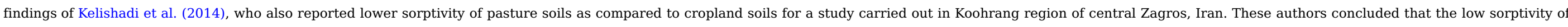
pastures was due to closure of pores and soil compaction by hoof trampling of grazing livestock which hinder movement of water in the topsoil (Kelishadi et al., 2014).

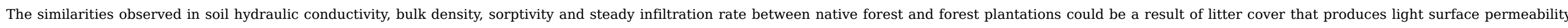

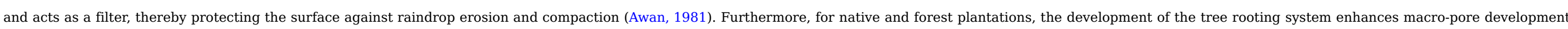
and maintenance.

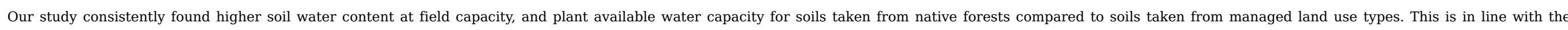

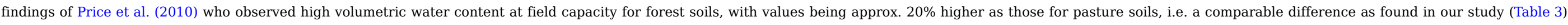

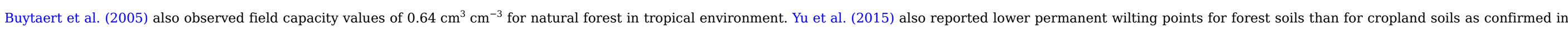
our study. Low plant available water capacity of soils under pastures was furthermore reported by Arnhold et al. (2015) who carried out their study in Lambwe Valley, Kenya.

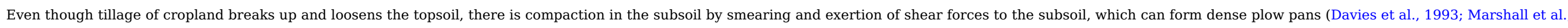

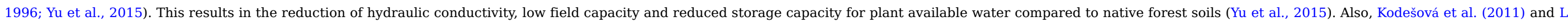

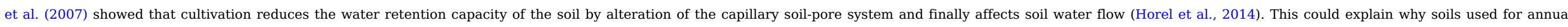

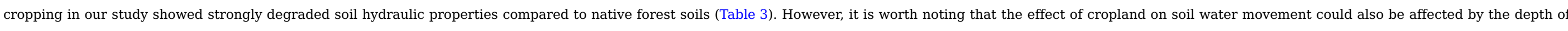
ploughing.

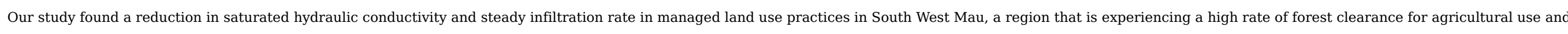

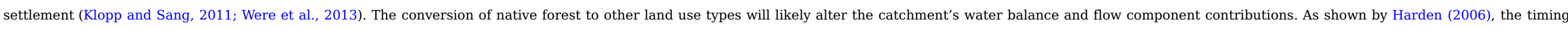

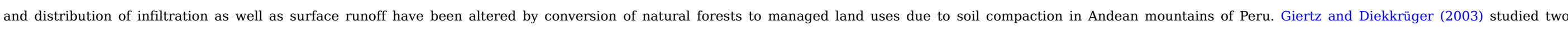

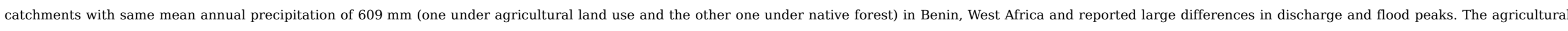

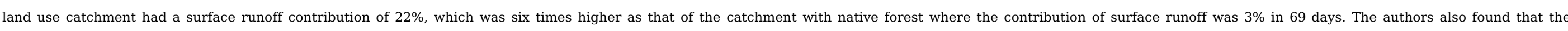

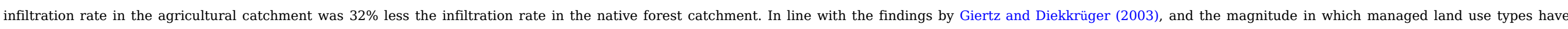

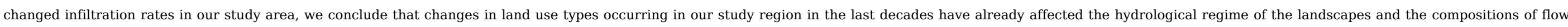
components.

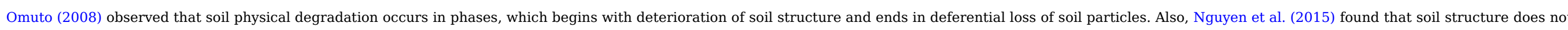

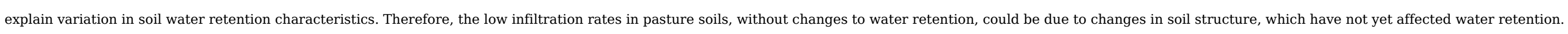

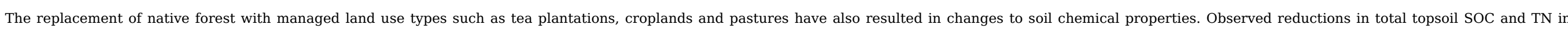

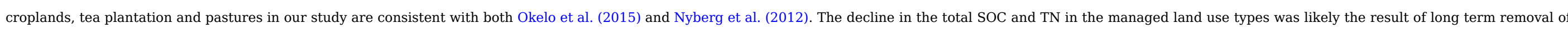

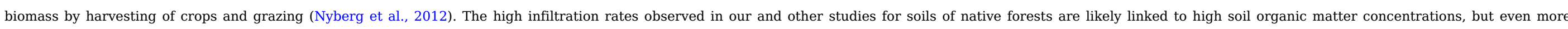

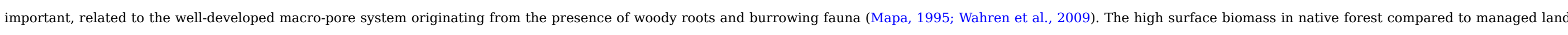

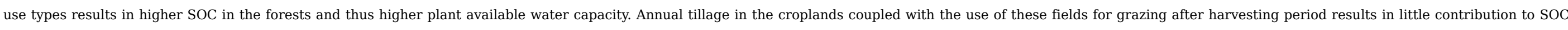
accumulation in these soils.

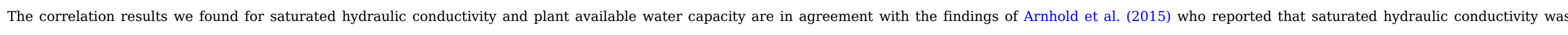

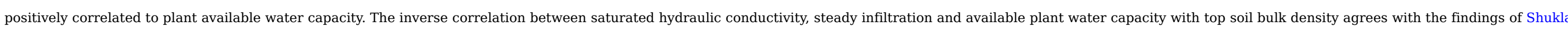

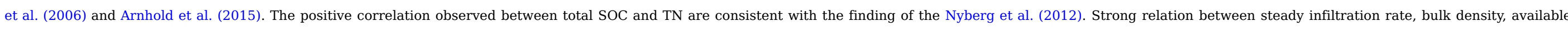
plant water capacity and total SOC (Table 5) shows that they are well suited for describing changes in soil hydraulic properties from native forest to managed land use types. 


\section{Conclusions}

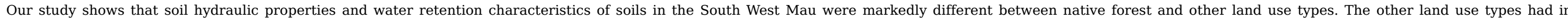

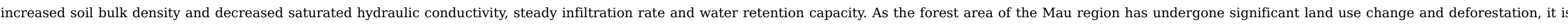

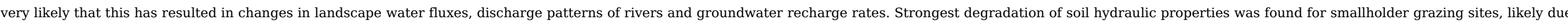

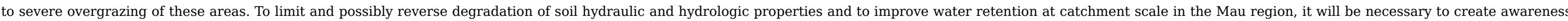
among stakeholders and to consider land use change effects on regional hydrology in regional land use planning

\section{Uncited references}

National Atlas of Kenya (1962) and Van Genuchten (1980).

\section{Acknowledgements}

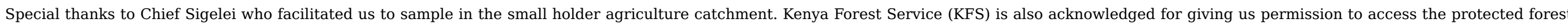

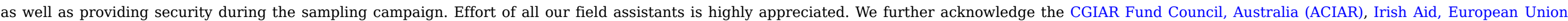

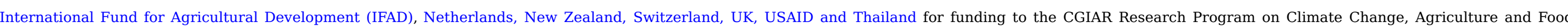
Security (CCAFS). L.B. would like to thank the DFG for supporting this work (BR2238/23-1)

\section{Appendix A. R code for calculating sorptivity and hydraulic conductivity from Philip's equation}

\# constructing data vectors by reading the.csv files where the data was stored.

infil_data <- read.csv ("D:/Mau/Nonlinear/C1P1.csv").

xdata <- infil data\$Reading.on.the.clock.

ydata <- infil data\$nfiltration.rate.cm.hr.

\# examining the data by plotting.

plot (xdata,ydata).

\# Fitting infiltration model (nonlinearly).

\#some starting values.

$\mathrm{S}=0$.

$\mathrm{K}=0$.

\#doing the fit.

fit_nonlin $=$ nls $($ ydata $\sim 0.5 * \mathrm{~S} *$ xdata $\wedge(-1 / 2)+\mathrm{K}$, start=list $(\mathrm{S}=\mathrm{S}, \mathrm{K}=\mathrm{K}))$

\#obtaining the fit values.

summary (fit_nonlin)

\section{References}

Akintoye O.A., Ukata S.U. and Esomonye H.I., The effects of landuse on the infiltration capacity of coastal plain soils of calabar-Nigeria, Int. J. Appl. Sci. Technol. 2 (2), 2012 , 80-84. 
Alegre J.C. and Cassel D.K., Dynamics of soil physical properties under alternative systems to slash-and-burn, Agric. Ecosyst. Environ. 58 (1), $1996,39-48$.

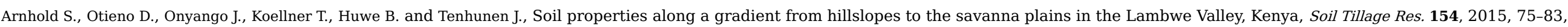
https://doi.org/10.1016/j.still.2015.06.021.

Awan N.M., Surface Water Hydrology vol. 1, 1981, National Book Foundation; Islamabad.

Batey T., Soil compaction and soil management - a review, Soil Use Manage. 25 (4), 2009, 335-345, https://doi.org/10.1111/j.1475-2743.2009.00236.x.

Binge F.W., Geology of the Kericho Area, Report No. 50, 1962, Geological Survey of Kenya.

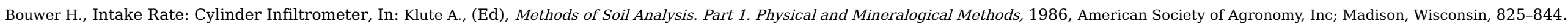

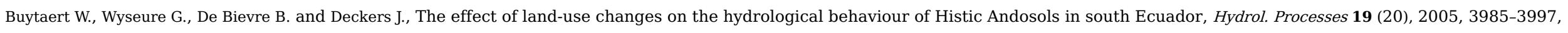

https://doi.org/10.1002/hyp.5867.

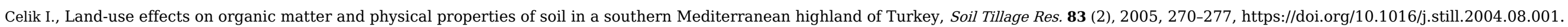

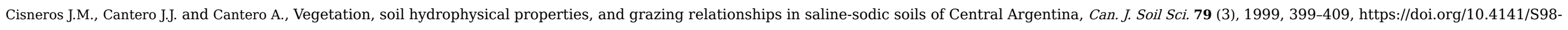
055.

Davies D.B., Eagle D.J. and Finney J.B., Soil Management, 5th ed., 1993, Farming Press; Ipswich.

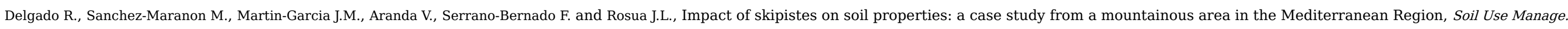

23, 2007, 269-277, https://doi.org/10.1111/j.1475-2743.2007.00093.x.

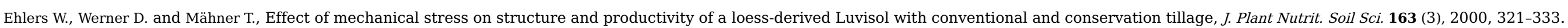

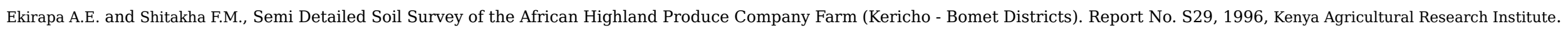

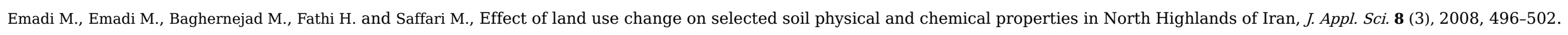

Eva H.D., Brink A. and Simonetti D., Monitoring Land Cover Dynamics in Sub-Saharan Africa, 2006, Institute for Environmental and Sustainability, Technical Report EUR, 22498.

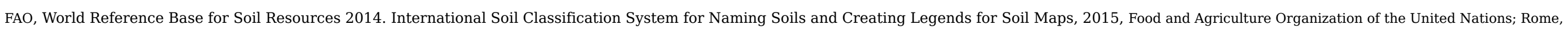
2015.

Fernandez E.S., Johnson Transformation, Version 1.4, 2015, R package, Available on https://cran.r-project.org/web/packages/Johnson/Johnson.pdf.

Gee G.W. and Bauder J.W., Particle-size analysis, In: Page A.L., (Ed), Methods of Soil Analysis. Part I. Physical and Mineralogical Methods, 2nd ed., 1986, Agronomy, 383-411.

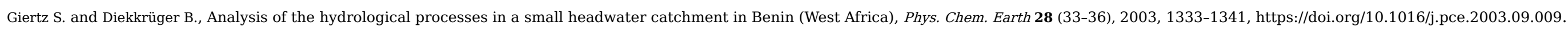

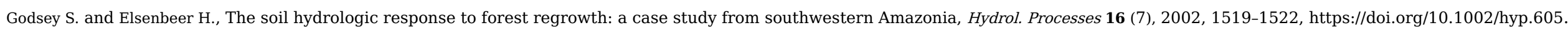

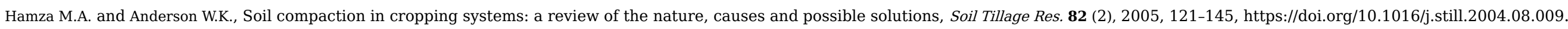

Harden C.P., Human impacts on headwater fluvial systems in the northern and central Andes, Geomorphology 79 (3), 2006, 249-263, https://doi.org/10.1016/j.geomorph.2006.06.021.

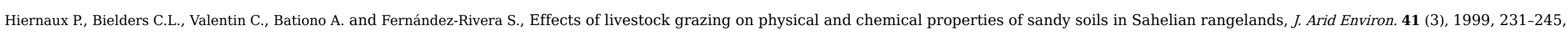

https://doi.org/10.1006/jare.1998.0475.

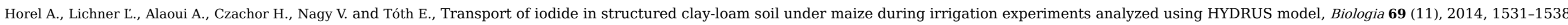




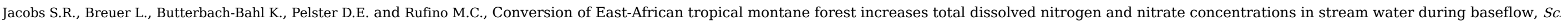
Total Environ. 2017, https://doi.org/10.1016/j.scitotenv.2017.06.100.

Jennings D.J., Geology of the Molo Area: Degree Sheet 42, 1971, Geological Survey of Kenya; N.E. Quarter.

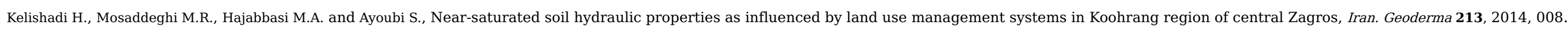
Kerfoot O., The vegetation of the South-West Mau forest, East Afr. Agric. For. J. 29, 1964, 295-318.

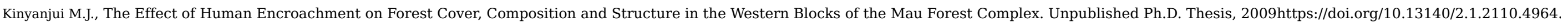
Klopp J.M. and Sang J.K., Maps, power, and the destruction of the Mau Forest in Kenya, Georget. J. Int. Aff. 2011, 125-134.

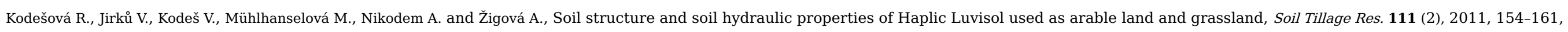
https://doi.org/10.1016/j.still.2010.09.007.

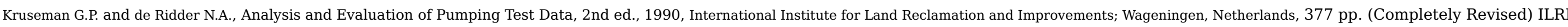
publication 47.

Lal R., Conversion of tropical rainforests: potential and ecological consequences, Adv. Agron. 39, 1986, 173-263.

Li X.G., Li F.M., Zed R., Zhan Z.Y. and Singh B., Soil physical properties and their relations to organic carbon pools as affected by land use in an alpine pastureland, Geoderma 139, 2007 , 98-105.

Mapa R.B., Effect of reforestation using Tectona grandis on infiltration and soil water retention, For. Ecol. Manage. 77 (1-3), 1995, 119-125.

Marshall T.J., Holmes J.W. and Rose C.W., Soil Physics, 3rd ed., 1996, Cambridge University Press; Cambridge.

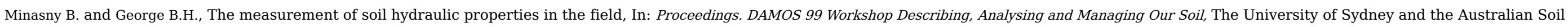
Science Society Inc. (NSW Branch), Sydney1999.

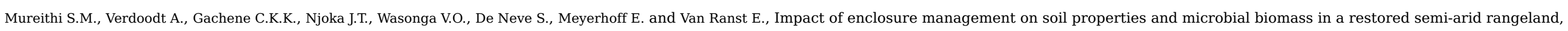
Kenya, J. Arid Land 6 (5), 2014, 561-570, https://doi.org/10.1007/s40333-014-0065-x.

National Atlas of Kenya, Soil Map, second ed., 1962, Survey of Kenya; Nairobi.

Nguyen P.M., De Pue J., Van Le K. and Cornelis W., Impact of regression methods on improved effects of soil structure on soil water retention estimates, J. Hydrol. 525, 2015, 598-606,

https://doi.org/10.1016/j.jhydrol.2015.04.014.

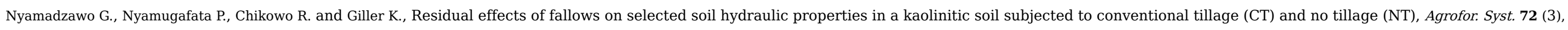
2008, 161-168, https://doi.org/10.1007/s10457-007-9057-6.

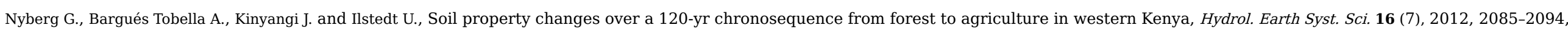
https://doi.org/10.5194/hess-16-2085-2012.

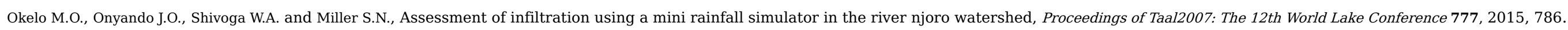

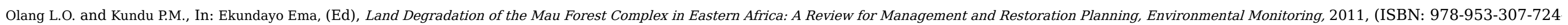

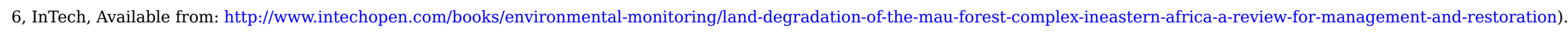

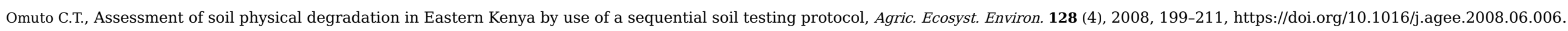




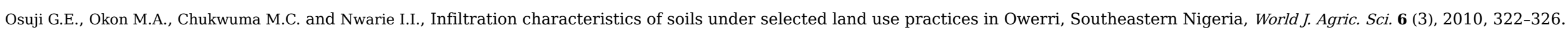

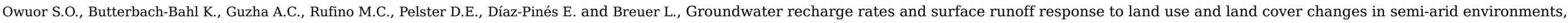
Ecol. Processes 5 (1), 2016, 16, https://doi.org/10.1186/s13717-016-0060-6.

Philip J.R., The theory of infiltration: I. The infiltration equation and its solutions, Soil Sci. 83, 1957, 345-357.

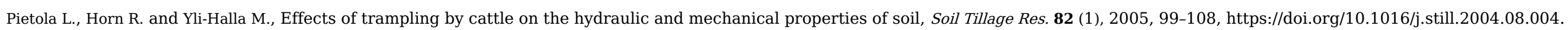

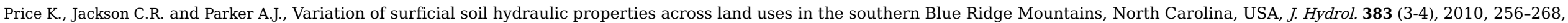
https://doi.org/10.1016/j.jhydrol.2009.12.041.

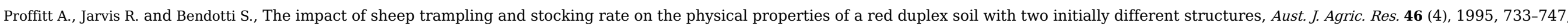
https://doi.org/10.1071/AR9950733.

R Core Team, R: A Language and Environment for Statistical Computing, 2015, R Foundation for Statistical Computing; Vienna, Austria https://www.R-project.org.

Reiners W.A., Bouwman A.F., Parsons W.F.J. and Keller M., Tropical rain forest conversion to pasture: changes in vegetation and soil properties, Ecol. Appl. 4 (2), $1994,363-377$.

Sarkar D. and Haldar A., Physical and Chemical Methods in Soil Analysis, 2005, New Age International Limited Publishers; New Delhi, 176 pp.

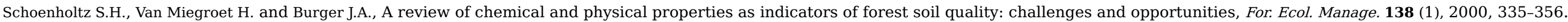

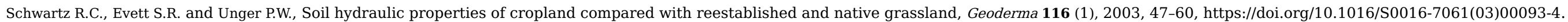

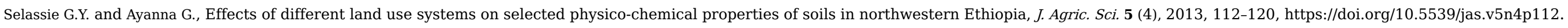

Shukla M.K., Lal R. and Ebinger M., Determining soil quality indicators by factor analysis, Soil Tillage Res. 87 (2), 2006, 194-204, https://doi.org/10.1016/j.still.2005.03.011.

Soil Survey Investigation Report No. 42, Kellog Soil Survey Laboratory Methods Manual, 2014 https://www.nrcs.usda.gov/Internet/FSE_DOCUMENTS/stelprdb1253871.pdf.

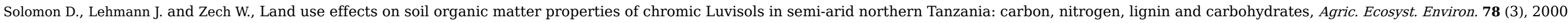
203-213.

Sombroek W.G., Braun H.M.H. and Van der Pouw B.J.A., Exploratory Soil Map and Agro-climatic Zone Map of Kenya, 1980. Scale 1: 1,000,000, 1980, Kenya Soil Survey.

Sterrett R.J., Groundwater and Wells, 3rd ed., 2007, Johnson screens; New Brighton, 812 pp.

Teixeira W.G., Sinclair F.L., Huwe B. and Schroth G., Soil water, In: Schroth G. and Sinclair F.L., (Eds.), Trees, Crops and Soil Fertility: Concepts and Research Methods, 2003, CABI Publishing, 209-234.

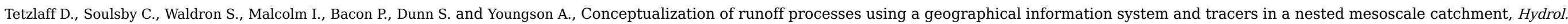
Processes 21 (10), 2007, 1289-1307, https://doi.org/10.1002/hyp.6309.

Van Genuchten M.Th., A closed-form equation for predicting the hydraulic conductivity of unsaturated soils, Soil Sci. Soc. Am. J. 44 (5), $1980,892-898$.

Wahren A., Feger K.H., Schwärzel K. and Münch A., Land-use effects on flood generation-considering soil hydraulic conductivity measurements in modeling, Adv. Geosci. 21, 2009 , 99-107.

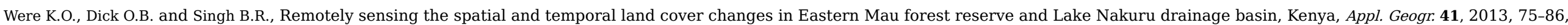

https://doi.org/10.1016/j.apgeog.2013.03.017.

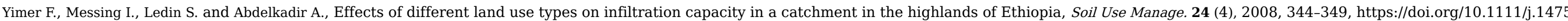
2743.2008.00182.x, (344-349, 24). 


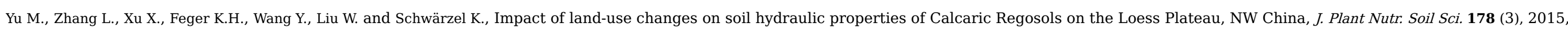
486-498, https://doi.org/10.1002/jpln.201400090.

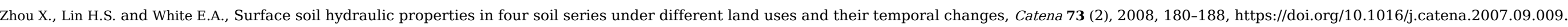

Zimmermann B., Elsenbeer H. and De Moraes J.M., The influence of land-use changes on soil hydraulic properties: implications for runoff generation, For. Ecol. Manage. 222 (1), 2006, 29-38,

https://doi.org/10.1016/j.foreco.2005.10.070

\section{Highlights}

- Effects of land use on soil hydrological properties in Mau Region, Kenya, assessed.

- Forest show approx. 2 times higher soil water infiltration as croplands/pastures.

- Land use intensification resulted in degradation of soil hydraulic properties.

\section{Queries and Answers}

Query: Please check the dochead and correct if necessary.

Answer: ok

Query: The author names have been tagged as given names and surnames (surnames are highlighted in teal color). Please confirm if they have been identified correctly.

Answer: Yes

Query: Please check Corresponding author address, and correct if necessary.

Answer: ok

Query: Please check the hierarchy of section headings and correct if necessary.

Answer: ok

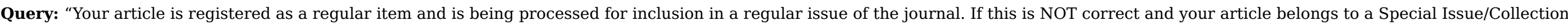
please contact s.ananthakrishnan@elsevier.com immediately prior to returning your corrections."

Answer: ok

Query: Please check the presentation of "Appendix A" and correct if necessary.

Answer: ok

Query: This section comprises references that occur in the reference list but not in the body of the text. Please cite each reference in the text or, alternatively, delete it.

Answer: please delete

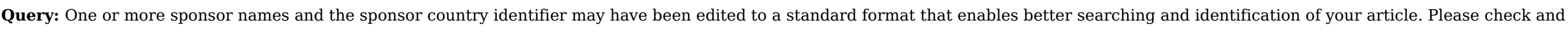
correct if necessary.

Answer: Yes 
Query: Please provide the volume number and page range in Ref. "Jacobs et al., 2017".

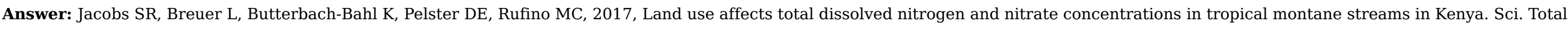
Environm. 603-604, 519-532

Query: Please check the presentation of tables and correct if necessary.

Answer: ok 